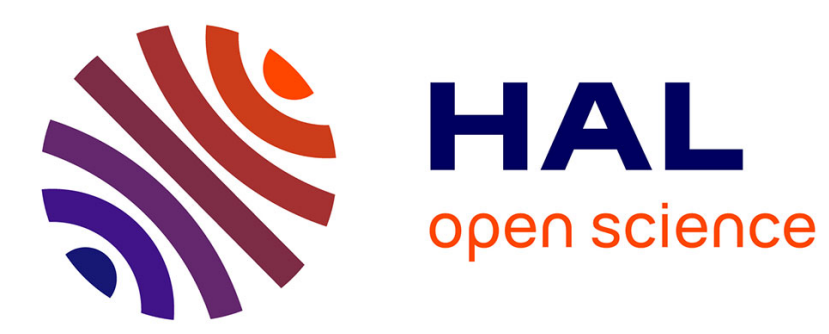

\title{
Bayesian BOLD and perfusion source separation and deconvolution from functional ASL imaging
}

Thomas Vincent, Florence Forbes, Philippe Ciuciu

\section{To cite this version:}

Thomas Vincent, Florence Forbes, Philippe Ciuciu. Bayesian BOLD and perfusion source separation and deconvolution from functional ASL imaging. ICASSP 2013 - IEEE International Conference on Acoustics, Speech, and Signal Processing, May 2013, Vancouver, Canada. pp.1003-1007, 10.1109/ICASSP.2013.6637800 . hal-00859373

\section{HAL Id: hal-00859373 \\ https://hal.inria.fr/hal-00859373}

Submitted on 7 Sep 2013

HAL is a multi-disciplinary open access archive for the deposit and dissemination of scientific research documents, whether they are published or not. The documents may come from teaching and research institutions in France or abroad, or from public or private research centers.
L'archive ouverte pluridisciplinaire HAL, est destinée au dépôt et à la diffusion de documents scientifiques de niveau recherche, publiés ou non, émanant des établissements d'enseignement et de recherche français ou étrangers, des laboratoires publics ou privés. 


\title{
BAYESIAN BOLD AND PERFUSION SOURCE SEPARATION AND DECONVOLUTION FROM FUNCTIONAL ASL IMAGING
}

\author{
Thomas Vincent ${ }^{1}$, Florence Forbes ${ }^{1}$, Philippe Ciuciu $^{2,3}$ \\ (1) INRIA, MISTIS, Grenoble University, LJK, Grenoble, France \\ ${ }^{(2)}$ CEA/DSV/I ${ }^{2}$ BM NeuroSpin center, Bât. 145, F-91191 Gif-sur-Yvette, France \\ (3) INRIA, Parietal, F-91893 Orsay, France.
}

\begin{abstract}
In many neuroscience applications, the Arterial Spin Labeling (ASL) fMRI modality arises as a preferable choice to the standard BOLD modality due to its ability to provide a quantitative measure of the Cerebral Blood Flow (CBF). Such a quantification is central but generally performed without consideration of a specific modeling of the perfusion component in the signal often handled via standard GLM approaches using the BOLD canonical response function as regressor. In this work, we propose a novel Bayesian hierarchical model of the ASL signal which allows activation detection and both the extraction of a perfusion and a hemodynamic component. Validation on synthetic and real data sets from event-related ASL show the ability of our model to address the source separation and double deconvolution problems inherent to ASL data analysis.
\end{abstract}

Index Terms- fMRI, ASL, deconvolution, Bayesian analysis, Monte Carlo Markov Chain inference.

\section{INTRODUCTION}

Functional MRI (fMRI) is the method of choice to noninvasively probe cerebral activity evoked by a set of controlled experimental conditions. A rising fMRI modality is Arterial Spin Labeling (ASL) which enables to quantify the cerebral perfusion, namely the cerebral blood flow (CBF) and emerges as a more direct biomarker of neuronal activity than the standard BOLD (Blood Oxygen Level Dependent) fMRI. The ASL modality is most commonly used as a static measure where the average perfusion is computed over a volume sequence lasting several minutes. Recently, ASL has been used in functional activation protocols $[1,2]$ and hence gives access to a dynamic measure of perfusion, namely the variations of CBF which are elicited by specific tasks.

ASL MRI mainly consists of acquiring pairs of control and label images and looking at the average control-label difference. The Signal-to-Noise Ratio (SNR) of this difference is very low so that several hundreds of image pairs need to be

Thanks to ARC AINSI (INRIA) for funding. acquired, thus increasing significantly the time spent by the subject in the scanner and making the acquisition very sensitive to the patient's movement. In addition, this averaging requires that the perfusion signal is at a steady state, limiting the scope of fMRI task experiments to baseline perfusion measurements or long block designs [3, 4]. In contrast, it is highly desirable to measure change in perfusion due to an effect of interest in activation paradigms from event-related designs. It is technically possible to collect event-related ASL data but most approaches to functional ASL data analysis use a standard linear model (GLM-based) formulation (eg. [5, 6]) with regressors encoding differences in control/tag scans and both ASL and BOLD activation signals being associated with the same canonical response function. The canonical hemodynamic response function (HRF) is generally used although it has been been calibrated on BOLD experiments only, thus reflecting simultaneous variations of $\mathrm{CBF}$, cerebral blood volume (CBV) and cerebral oxygen consumption $\left(\mathrm{CMRO}_{2}\right)$. In contrast, the perfusion signal only reflects variation in $\mathrm{CBF}$ so that the associated response, that we will call the perfusion response function (PRF) hereafter, is likely to differ from the HRF. Here, we propose to recover both a hemodynamic (BRF for BOLD response function) and a perfusion (PRF) response functions from event-related functional ASL data. We make use of a joint detection estimation (JDE) formalism introduced in $[7,8]$. In the BOLD context, the JDE framework has proven to successfully extract the HRF while also performing activation detection. We extend this formalism to model an additional perfusion component linked to the BOLD one through a common activation detection. So doing, the hope is to provide a better estimation of perfusion modulation effects, which can then be translated into more accurate quantitative measures. Indeed, a lot of work in ASL analysis focuses on perfusion quantification issues $[9,10]$ without consideration of a perfusion response modeling although this would benefit a better effect estimation. Note however that perfusion quantification per se is out of the scope of this paper and will be addressed in future work. After introducing our ASL generative model in Sections 2-3, we give some details on its estimation in Section 4 and validate our approach on both artificial and 
real data sets in Section 5.

\section{ASL GENERATIVE MODEL}

Following [7, 8], the brain is assumed to have been partitioned into several functional homogeneous parcels, each of which gathers signals which share the same response shapes. The question of how to obtain such a parcellation is beyond the scope of this paper and has been addressed in $[8,11]$. Here, for the sake of conciseness we only consider a single parcel denoted by $\mathcal{P}$. At voxel $j \in \mathcal{P}$ with $|\mathcal{P}|=J$, the ASL time series $\boldsymbol{y}_{j}$ is measured at times $\left(t_{n}\right)_{n=1: N}$ where $t_{n}=n \mathrm{TR}$, $N$ is the number of scans and TR the time of repetition. The ASL signal is modelled as a linear combination of BOLD and perfusion components, the former remaining the same as the one proposed in [8], while a bilinear and time-invariant system is added to account for the perfusion component. The overall generative model reads $\forall j \in \mathcal{P}$ :

$$
\boldsymbol{y}_{j}=\sum_{m=1}^{M}\left[\boldsymbol{c}_{j}^{m} \boldsymbol{W} \boldsymbol{X}^{m} \boldsymbol{g}+\boldsymbol{a}_{j}^{m} \boldsymbol{X}^{m} \boldsymbol{h}\right]+\boldsymbol{P} \boldsymbol{\ell}_{j}+\alpha_{j} \boldsymbol{w}+\boldsymbol{b}_{j},
$$

where the perfusion and BOLD components are given by the two terms in Eq. (1). Vectors $\boldsymbol{h} \in \mathbb{R}^{D+1}$ and $\boldsymbol{g} \in \mathbb{R}^{D+1}$ represent the unknown BRF and PRF shapes in $\mathcal{P}$, respectively. For the sake of simplicity, the temporal resolution of the BOLD and perfusion response components are the same, without loss of generality. Shapes $\boldsymbol{h}$ and $\boldsymbol{g}$ are constant within parcel $\mathcal{P}$ but the corresponding response levels $a_{j}^{m}$ and $c_{j}^{m}$, which model the magnitude of activation, may vary in space and across experimental conditions. We denote the BOLD response levels (BRLs) by $\boldsymbol{A}=\left\{\boldsymbol{a}^{m}, m=1: M\right\}$ with $\boldsymbol{a}^{m}=\left\{a_{j}^{m}, j \in \mathcal{P}\right\}, a_{j}^{m}$ being the response level at voxel $j$ for condition $m$. Similarly, perfusion response levels (PRLs) are denoted by $\boldsymbol{C}=\left\{\boldsymbol{c}^{m}, m=1: M\right\}$ with $c^{m}=\left\{c_{j}^{m}, j \in \mathcal{P}\right\} . \boldsymbol{X}^{m}$ denotes the $N \times(D+1)$ binary matrix $\boldsymbol{X}^{m}=\left\{x_{m}^{n-d \Delta t}, n=1: N, d=0: D\right\}$ that encodes the arrival times of the stimulus occurrences for the $m$ th experimental condition, with $\Delta t<T R$ being the sampling period of the unknown response functions (PRF and BRF). Vector $\boldsymbol{w}$ is $N$-dimensional such that $w_{t_{n}}=1$ if $t_{n}$ is even (control volume) and $w_{t_{n}}=-1$ otherwise (tagged volume) and $\boldsymbol{W}=\operatorname{diag}(\boldsymbol{w})$ is the diagonal matrix with $\boldsymbol{w}$ as diagonal components. More precisely, $\boldsymbol{w}$ encodes the difference in magnetization signs between control and tagged ASL volumes. Matrix $\boldsymbol{P}=\left[\boldsymbol{p}_{1}, \ldots, \boldsymbol{p}_{O}\right]$ of size $N \times O$ comprises the values at times $t_{n}$ of an orthonormal basis (i.e., $\boldsymbol{P}^{\mathrm{t}} \boldsymbol{P}=\boldsymbol{I}_{O}$ ) consisting of $O$ functions $\boldsymbol{p}_{o}=\left(p_{o, t_{n}}, n=1: N\right)^{\mathrm{t}}$ that take a potential drift and any other nuisance effect (eg. slow motion parameters) into account. Vector $\ell_{j}=\left(\ell_{o, j}, o=1: O\right)^{\mathrm{t}}$ contains the corresponding unknown regression coefficients for voxel $j$. Finally, the scalar $\alpha_{j}$ models the perfusion baseline at voxel $j$. For the sake of simplicity, a white Gaussian noise $\boldsymbol{b}_{j} \sim \mathcal{N}\left(\mathbf{0}, v_{b_{j}} \boldsymbol{I}_{N}\right)$ is considered here whereas a straight- forward extension to $\mathrm{AR}(1)$ noise process can be derived using $[8,12]$.

\section{PERFUSION AND HEMODYNAMIC PRIORS}

Response shapes. Following [8], the BRF and PRF shapes are assumed to follow a prior multivariate Gaussian distribution whose covariance matrix $\Sigma$ embodies a constraint on the second order derivative so as to account for temporal smoothness. As this modeling remains non-parametric, any shape is allowed and it is worth noting that the two BRF and PRF shapes may differ. Formally, these priors read $\boldsymbol{h} \sim \mathcal{N}\left(0, v_{\boldsymbol{h}} \Sigma\right)$ and $\boldsymbol{g} \sim \mathcal{N}\left(0, v_{\boldsymbol{g}} \Sigma\right)$, where $v_{\boldsymbol{h}}$ and $v_{\boldsymbol{g}}$ are unknown variances.

Response Levels. For a given experimental condition $m$, the BOLD (BRLs) and perfusion (PRLs) response levels are assumed to follow spatial Gaussian mixture models with different means and variances but governed by the same hidden Markov random field encoding voxels activation states. Indeed, the actual BOLD and perfusion response levels are likely to differ as they do not reflect the same physiological measure: $\mathrm{CBF}$ vs. a combination of $\mathrm{CBV}, \mathrm{CBF}$ and $\mathrm{CMRO}_{2}$. However, the activation states are assumed to be the same for both response levels. The aim is to model a coupling between the perfusion and BOLD components, allowing the extraction of the perfusion component by taking advantage of the higher SNR in the BOLD component. In order to encode the activation states, class assignment variables $\boldsymbol{Q}=$ $\left\{\boldsymbol{q}^{m}, m=1: M\right\}$ are introduced where $\boldsymbol{q}^{m}=\left\{q_{j}^{m}, j \in \mathcal{P}\right\}$ and $q_{j}^{m}$ represents the activation class at voxel $j$ for condition $m$. We consider here two classes, for activated $(i=2)$ and non-activated $(i=1)$ voxels. Formally, the BRLs and PRLs are a priori independent conditionally to $Q$ :

$p(\boldsymbol{A}, \boldsymbol{C} \mid \boldsymbol{Q})=\prod_{m=1}^{M} \prod_{i=1}^{2} \prod_{j=1}^{J_{i}^{m}}\left[\mathcal{N}\left(a_{j}^{m} ; \mu_{i}^{m}, v_{i}^{m}\right) \mathcal{N}\left(c_{j}^{m} ; \eta_{i}^{m}, \rho_{i}^{m}\right)\right]$

with $J_{i}^{m}=\left\{j \in J \mid q_{j}^{m}=i\right\}$ and $\mu_{i}^{m}, v_{i}^{m}$ and $\eta_{i}^{m}, \rho_{i}^{m}$ are the unknown means and variances of the $i^{\text {th }}$ mixture component, for the BRLs and PRLs, respectively. As in [8], spatial correlation is introduced through Ising models on each $\boldsymbol{q}^{m}$ :

$$
p\left(\boldsymbol{q}^{m} \mid \beta_{m}\right)=Z\left(\beta_{m}\right)^{-1} \exp \left(\beta_{m} \sum_{j \sim j^{\prime}} \mathbb{1}\left(q_{j}^{m}=q_{j^{\prime}}^{m}\right)\right)
$$

where $j \sim j^{\prime}$ indicates that the sum extends over all neighboring pairs of voxels. We denote $\mathbb{1}(A)=1$ if $A$ is true and $\mathbb{1}(A)=0$ otherwise. $\beta_{m}$ is the spatial regularization factor and $Z\left(\beta_{m}\right)$ is the partition function.

Perfusion baseline. A priori, this quantity should not be difficult to extract as it roughly corresponds to the mean over the differences between control and tagged volumes. Hence, a simple Gaussian prior is introduced: $\forall j, \alpha_{j} \sim \mathcal{N}\left(0, v_{\boldsymbol{\alpha}}\right)$ and $\boldsymbol{\alpha}=\left\{\alpha_{j}, j \in \mathcal{P}\right\}$. 
Drift coefficients. The prior on these coefficients $\boldsymbol{L}=$ $\left\{\boldsymbol{\ell}_{j}, j \in \mathcal{P}\right\}$ is Gaussian: $\forall j, \boldsymbol{\ell}_{j} \sim \mathcal{N}\left(\mathbf{0}, v_{\ell} \boldsymbol{I}_{O}\right)$; see [8] for further details.

Hyper-parameters. Akin to [8], non-informative Jeffrey priors are adopted for $\left\{v_{\boldsymbol{b}}, v_{\boldsymbol{\ell}}, v_{\boldsymbol{\alpha}}, v_{\boldsymbol{g}}, v_{\boldsymbol{h}}\right\}$ and proper conjugate priors are considered for the mixture parameters of BRLs and PRLs $\boldsymbol{\theta}_{\boldsymbol{A}}=\left\{\mu_{i}^{m}, v_{i}^{m}, i=1: 2, m=1: M\right\}$ and $\boldsymbol{\theta}_{\boldsymbol{C}}=\left\{\eta_{i}^{m}, \rho_{i}^{m}, i=1: 2, m=1: M\right\}$. Positive spatial correlation parameters $\boldsymbol{\beta}=\left\{\beta_{m}, m=1: M\right\}$ are associated with a uniform prior over $[0,1.5]$ as values over 1.5 correspond to completely correlated fields [8]. All hyperparameters are grouped in the set $\Theta$.

\section{BAYESIAN MCMC INFERENCE}

In [8], for ease of computation we have introduced the hidden assignment variables $\boldsymbol{Q}$ into the derivation and the sampling procedure of the joint posterior distribution. Here, we push further this idea considering the prior independence of $\boldsymbol{A}$ and $C$ conditionally to $Q$ :

$$
\begin{array}{r}
p(\boldsymbol{A}, \boldsymbol{C}, \boldsymbol{Q}, \boldsymbol{h}, \boldsymbol{g}, \boldsymbol{L}, \boldsymbol{\alpha}, \boldsymbol{\Theta} \mid \boldsymbol{y}) \propto p\left(\boldsymbol{y} \mid \boldsymbol{A}, \boldsymbol{C}, \boldsymbol{h}, \boldsymbol{g}, \boldsymbol{L}, \boldsymbol{\alpha}, v_{\boldsymbol{b}}\right) \\
\ldots \times p\left(\boldsymbol{A} \mid \boldsymbol{Q}, \boldsymbol{\theta}_{\boldsymbol{A}}\right) p\left(\boldsymbol{C} \mid \boldsymbol{Q}, \boldsymbol{\theta}_{\boldsymbol{C}}\right) p(\boldsymbol{Q} \mid \boldsymbol{\beta}) p\left(\boldsymbol{h} \mid v_{\boldsymbol{h}}\right) p\left(\boldsymbol{g} \mid v_{\boldsymbol{g}}\right) \\
\ldots \times p\left(\boldsymbol{\alpha} \mid v_{\boldsymbol{\alpha}}\right) p\left(\boldsymbol{L} \mid v_{\ell}\right) p(\boldsymbol{\Theta})
\end{array}
$$

Each variable $x \in \mathcal{X}(\mathcal{X}=\{\boldsymbol{A}, \boldsymbol{Q}, \boldsymbol{h}, \boldsymbol{g}, \boldsymbol{\alpha}, \boldsymbol{L}, \boldsymbol{\Theta}\})$ is sampled using a hybrid Metropolis-Gibbs sampling scheme and posterior mean (PM) estimates are computed from the samples according to: $\widehat{x}^{\mathrm{PM}}=\sum_{k=K_{0}}^{K_{1}} x^{(k)} / K, \quad \forall x \in \mathcal{X}$ where $K=K_{1}-K_{0}+1$ and $K_{0}$ stands for the length of the burn-in period (see [8] for details).

Posterior conditional distributions $p\left(x^{k} \mid(\mathcal{X} \backslash x)^{k-1}, \boldsymbol{y}\right)$ computed at each iteration $k$ of the sampling scheme are comparable to those derived in [8], as the formulations for the variables involved in the perfusion component can be obtained from the ones of the BOLD component by mainly replacing $\boldsymbol{X}$ with $\boldsymbol{W} \boldsymbol{X}$. Next, we only provide the conditional posterior distributions that substantially differ from [8]. For the sake of conciseness, iteration index $k$ is dropped in the following formulas.

Assignment variables. The full conditional posterior of the $\boldsymbol{q}^{m}$ is identified to an asymmetric Ising field:

$p\left(q_{j}^{m} \mid \mathcal{X} \backslash q_{j}^{m}\right) \propto \exp \left[\beta^{m} U\left(\kappa_{j}\right)+\sum_{i=1}^{2} \mathbb{1}\left(q_{j}^{m}=i\right) S_{i, j}^{m}\right]$

with: $U\left(\kappa_{j}\right)=\sum_{j^{\prime} \in \kappa_{j}} \mathbb{1}\left(q_{j}^{m}=q_{j^{\prime}}^{m}\right), \kappa_{j}=\{$ neighbors of $j\}$, and $S_{i, j}^{m}=\left(-\frac{\ln \left(v_{i}^{m} \rho_{i}^{m}\right)}{2}-\frac{\left(a_{j}^{m}-\mu_{i}^{m}\right)^{2}}{2 v_{i}^{m}}-\frac{\left(c_{j}^{m}-\eta_{i}^{m}\right)^{2}}{2 \rho_{i}^{m}}\right)$.

BRLs and PRLs. Let us introduce the following notations for partial residual quantities: $\overline{\boldsymbol{y}}_{j}=\boldsymbol{y}_{j}-P \boldsymbol{\ell}_{j}-\alpha_{j} \boldsymbol{w}, \widehat{\boldsymbol{y}}_{j}^{\prime m}=$ $\overline{\boldsymbol{y}}_{j}-\sum_{m^{\prime} \neq m} a_{j}^{m^{\prime}} \boldsymbol{X}^{m^{\prime}} \boldsymbol{h}, \check{\boldsymbol{y}}_{j}^{\backslash m}=\overline{\boldsymbol{y}}_{j}-\sum_{m^{\prime} \neq m} c_{j}^{m^{\prime}} \boldsymbol{W} \boldsymbol{X}^{m^{\prime}} \boldsymbol{g}$.
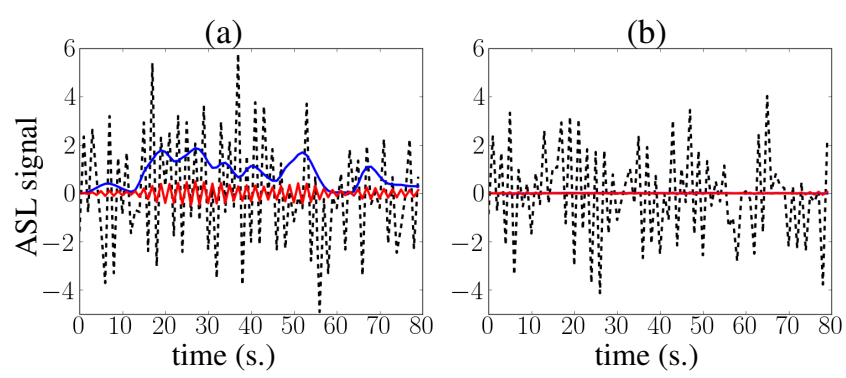

Fig. 1. Illustration of artificial data signals. The perfusion and BOLD components are depicted in red and blue, respectively. The final ASL signal (with drifts, noise and perfusion baseline) is depicted in dashed black lines. (a) Activating voxel. (b) Non-activating voxel. (a)

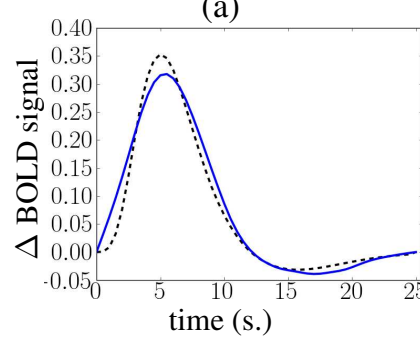

(b)

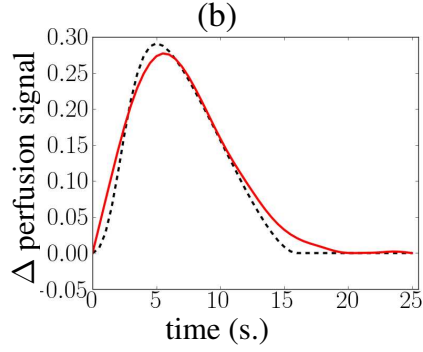

Fig. 2. Results on artificial data for response functions. Groundtruth response curves are depicted in black dashed lines and estimated responses in colored continuous lines. (a) BOLD response functions. (b) Perfusion response functions.

The BRLs and PRLs follow the full conditional posterior distributions given by:

$$
\begin{array}{r}
\left(a_{j}^{m} \mid q_{j}^{m}=i, \ldots\right) \sim \mathcal{N}\left(\frac{\boldsymbol{\epsilon}_{m}^{T} \hat{\boldsymbol{y}}_{j}^{\backslash m}+v_{b_{j}} \mu_{i}^{m}}{\left(v_{i, j}^{m}\right)^{-1} v_{b_{j}} v_{i}^{m}}, v_{i, j}^{m}\right), \\
\text { with } \boldsymbol{\epsilon}_{m}=\left(\boldsymbol{X}^{m}\right)^{T} \boldsymbol{h} \text { and } v_{i, j}^{m}=\frac{v_{b_{j}} v_{i}^{m}}{\boldsymbol{\epsilon}_{m}^{T} \boldsymbol{\epsilon}_{m} v_{i}^{m}+v_{b_{j}}}, \\
\left(c_{j}^{m} \mid q_{j}^{m}=i, \ldots\right) \sim \mathcal{N}\left(\frac{\boldsymbol{\zeta}_{m}^{T} \breve{\boldsymbol{y}}_{j}^{\backslash m}+v_{b_{j}} \eta_{i}^{m}}{\left(\rho_{i, j}^{m}\right)^{-1} v_{b_{j}} \rho_{i}^{m}}, \rho_{i, j}^{m}\right), \\
\text { with } \boldsymbol{\zeta}_{m}=\boldsymbol{W}\left(\boldsymbol{X}^{m}\right)^{T} \boldsymbol{g} \text { and } \rho_{i, j}^{m}=\frac{v_{b_{j}} \rho_{i}^{m}}{\boldsymbol{\zeta}_{m}^{T} \boldsymbol{\zeta}_{m} \rho_{i}^{m}+v_{b_{j}}} .
\end{array}
$$

Perfusion baseline. The partial residual quantity involved here is denoted: $\tilde{\boldsymbol{y}}_{j}=\boldsymbol{y}_{j}-\sum_{m=1}^{M}\left(a_{j}^{m} \boldsymbol{X}^{m} \boldsymbol{h}-c_{j}^{m} \boldsymbol{W} \boldsymbol{X}^{m} \boldsymbol{g}\right)-$ $P \ell_{j}-\alpha_{j} \boldsymbol{w}$. The full conditional posterior distribution of the perfusion baseline reads:

$$
\left(\alpha_{j} \mid \mathcal{X} \backslash \alpha_{j}, \boldsymbol{y}_{j}\right) \sim \mathcal{N}\left(\frac{v_{\boldsymbol{\alpha}} \boldsymbol{W}^{T} \tilde{\boldsymbol{y}}_{j}}{N v_{\boldsymbol{\alpha}}+v_{b_{j}}}, \frac{v_{\boldsymbol{\alpha}} v_{b_{j}}}{N v_{\boldsymbol{\alpha}}+v_{b_{j}}}\right)
$$

\section{RESULTS}

\subsection{Artificial data}

The ASL artificial 4D signals are simulated with a realistic low SNR according to the observation model in Eq. (1) 
where $\boldsymbol{h}$ and $\boldsymbol{g}$ are depicted in Fig.2 (black dashed lines), $\boldsymbol{P}$ is a polynomial basis of order 4 and $\boldsymbol{X}^{m}$ encodes a fast event-related paradigm comprising two conditions (ISI $=3.5$ s.). Drift coefficients are drawn as $\ell_{j} \sim \mathcal{N}\left(0,10 . I_{O}\right)$ and noise realizations according to $\boldsymbol{b}_{j} \sim \mathcal{N}\left(0,2 . I_{N}\right)$. BOLD response levels are drawn according to: $\left(a_{j}^{m} \mid q_{j}^{m}=2\right) \sim$ $\mathcal{N}(2.2,0.3)$ (activating class) and $\left(a_{j}^{m} \mid q_{j}^{m}=1\right) \sim \mathcal{N}(0,0.3)$ (inactivating class). To be realistic, perfusion response levels are generated with a lower contrast than BRLs: $\left(c_{j}^{m} \mid q_{j}^{m}=\right.$ 2) $\sim \mathcal{N}(1.6,0.3)$ and $\left(c_{j}^{m} \mid q_{j}^{m}=1\right) \sim \mathcal{N}(0,0.3)$. Activation states (assignment variables $\boldsymbol{Q}$ ) are set by hand-drawn maps, an illustration of which is shown on the top-right map of Fig. 3 for $m=1$. Illustrations of the generated time-series are depicted in Fig. 1. In what follows, results only concern condition $m=1$ as the same conclusions hold for $m=2$.

Results on response estimates are shown in Fig. 2 and state the quality of the deconvolution performed by our approach which is able to distinguish the two different response profiles. Some slight inaccuracies are observed in the tail part, which is indeed more difficult to estimate as it is associated with a very low signal strength. Response level and label estimates shown in Fig. 3 also indicate that our Bayesian approach performs well in activation detection, where activating clusters are well recovered.
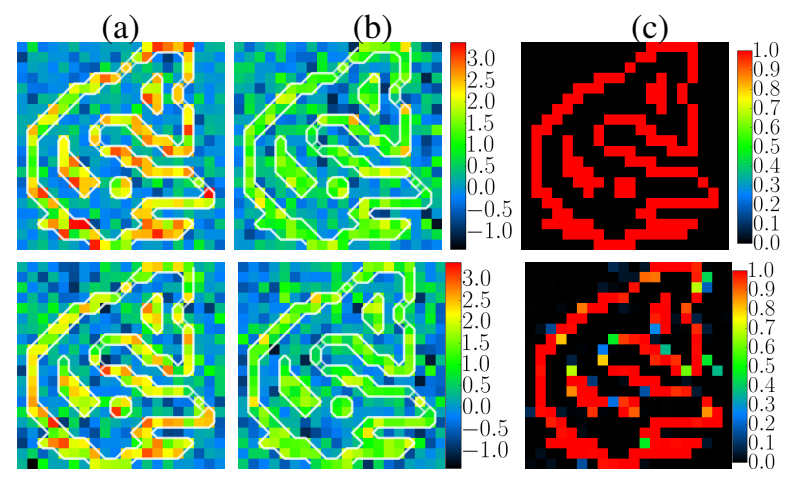

Fig. 3. Results on artificial data for response levels. Top row: ground-truth maps. Bottom row: estimated maps. (a) BOLD response levels. (b) Perfusion response levels. Superimposed white lines delineate the ground-truth activated cluster. (c) Assignment variables (activation states).

\subsection{Real data}

Real ASL data were recorded during an experiment designed to map auditive and visual brain functions, which consisted of $N=291$ scans lasting $T R=3 s$., each yielding a 3-D volume composed of $64 \times 64 \times 22$ voxels (resolution of $3 \times 3 \times$ $3.5 \mathrm{~mm}$ ). The paradigm was fast event-related (mean ISI=5.1 s.) comprising sixty auditive and visual stimuli. A region of interest in the right temporal lobe was defined manually.

Fig. 5 depicts the response estimates superimposed to the canonical HRF shape and we can clearly state the departure from the latter, especially in the peak duration which is shorter (a)

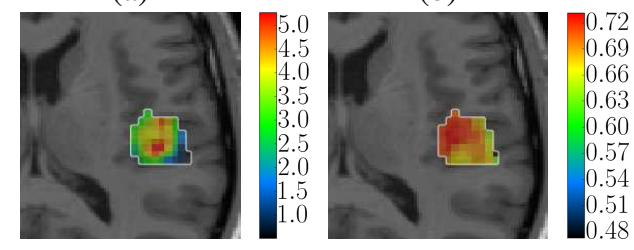

Fig. 4. Response levels estimation results on real data (zoomed on a right temporal region). (a): BOLD response level estimates. (b): Perfusion response level estimates. (a)

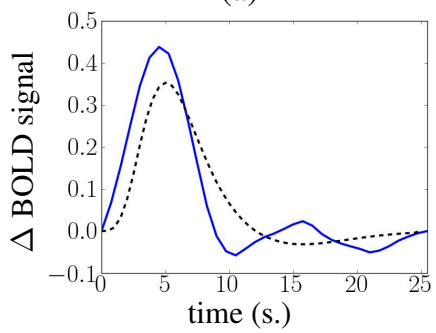

(b)

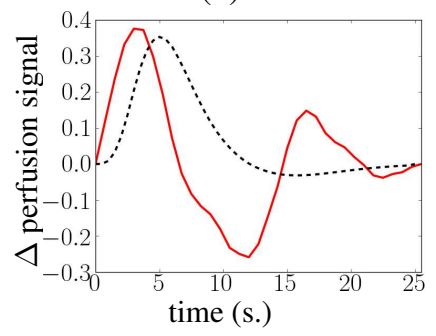

Fig. 5. Response estimation results on real data. (a): BOLD response estimate. (b): Perfusion response estimate. The canonical HRF is depicted as a black dashed line.

( $7 \mathrm{~s}$. for BOLD response vs. $10 \mathrm{~s}$. for the canonical response). The first $10 \mathrm{~s}$. of the estimated perfusion response in Fig. 5(b) are interpretable with a time-to-peak earlier than the canonical one ( $4 \mathrm{~s}$. vs $5 \mathrm{~s}$.). The tail part of this response is yet difficult to interpret and might represent a subsequent recurring activation due to overlapping responses. On Fig. 4, the spatial support of activations seem roughly the same between the BOLD and perfusion components but they do not precisely match so that the coupling constraint might have to be relaxed. Still, the relative magnitude of the estimated effects is sensible with a ratio $\mathrm{PRL} / \mathrm{BRL} \approx 1 / 5$ which is consistent with the known scale of the BOLD (5\% of signal variation) and perfusion effect (1\% of signal variation).

\section{CONCLUSION}

The characterization of the perfusion response function from ASL data in the context of an event-related paradigm is a challenging issue as the perfusion component suffers from a low SNR compared to the BOLD component. The Bayesian hierarchical approach presented here is able to perform BOLD and perfusion source separation and provide first insights of the perfusion response whose peak appeared earlier than the BOLD response. Still, the obtained results for the perfusion profile are not fully interpretable, especially its return to baseline and future work will focus on improving the temporal model by encoding regularizing relations between events for example, as in [13]. Finally, a quantification method will be incorporated so as properly relate the variations observed in the recovered PRFs with the underlying CBF. 


\section{REFERENCES}

[1] H. Raoult, J. Petr, E. Bannier, A. Stamm, J-Y. Gauvrit, C. Barillot, and J-C. Ferré, "Arterial spin labeling for motor activation mapping at $3 \mathrm{~T}$ with a 32-channel coil: reproducibility and spatial accuracy in comparison with BOLD fMRI.," Neuroimage, vol. 58, no. 1, pp. 157-67, Sep. 2011.

[2] H. Raoult, J-C. Ferré, J. Petr, E. Bannier, A. Stamm, C. Barillot, and J-Y. Gauvrit, "Functional arterial spin labeling: Optimal sequence duration for motor activation mapping in clinical practice.," Journal of magnetic resonance imaging, vol. 36, no. 6, pp. 1435-44, Dec. 2012.

[3] J. Detre, J. Wang, Z. Wang, and H. Rao, “Arterial spin-labeled perfusion MRI in basic and clinical neuroscience," Curr Opin Neurol, vol. 22, pp. 348-355, 2009.

[4] K. Restom, K. J. Bangen, M. W. Bondi, J. E. Perthen, and T. T. Liu, "Cerebral blood flow and BOLD responses to a memory encoding task: a comparison between healthy young and elderly adults," Neuroimage, vol. 37, pp. 430-439, 2007.

[5] J. A. Mumford, L. Hernandez-Garcia, G. R Lee, and T. E. Nichols, "Estimation efficiency and statistical power in arterial spin labeling fMRI," Neuroimage, vol. 33, pp. 103-114, 2006.

[6] L. Hernandez-Garcia, H. Jahanian, and D. B. Rowe, "Quantitative analysis of arterial spin labeling FMRI data using a general linear model," Magnetic Resonance Imaging, vol. 28, no. 7, pp. 919-927, 2010.

[7] S. Makni, J. Idier, T. Vincent, B. Thirion, G. DehaeneLambertz, and P. Ciuciu, "A fully Bayesian approach to the parcel-based detection-estimation of brain activity in fMRI," Neuroimage, vol. 41, no. 3, pp. 941-969, July 2008.

[8] T. Vincent, L. Risser, and P. Ciuciu, "Spatially adaptive mixture modeling for analysis of within-subject fMRI time series," IEEE Trans. Med. Imag., vol. 29, no. 4, pp. 1059-1074, Apr. 2010.

[9] M.W. Woolrich, P. Chiarelli, D. Gallichan, J. Perthen, and T.T. Liu, "Bayesian inference of hemodynamic changes in functional arterial spin labeling data," Magn Reson Med., vol. 56, no. 4, pp. 891-906, 2006.

[10] W-C. Wu, K. S. St Lawrence, D. J. Licht, and D. Wang, "Quantification Issues in Arterial Spin Labeling Perfusion Magnetic Resonance Imaging," Topics in Magnetic Resonance Imaging, vol. 21, no. 2, pp. 65-73, 2010.
[11] B. Thirion, G. Flandin, P. Pinel, A. Roche, P. Ciuciu, and J.-B. Poline, "Dealing with the shortcomings of spatial normalization: Multi-subject parcellation of fMRI datasets," Hum. Brain Mapp., vol. 27, no. 8, pp. 678693, Aug. 2006.

[12] L. Chaari, T. Vincent, F. Forbes, M. Dojat, and P. Ciuciu, "Fast joint detection-estimation of evoked brain activity in event-related fMRI using a variational approach," IEEE trans. Med. Imag., 10.1109/TMI.2012.2225636, to appear.

[13] P. Ciuciu, S. Sockeel, T. Vincent, and J. Idier, "Modelling the neurovascular habituation effect on fMRI time series," in 34th Proc. IEEE ICASSP, Taipei, Taiwan, Apr. 2009, pp. 433-436. 